\title{
Journal of

\section{Non-leaching antimicrobial surfaces through polydopamine bio-inspired coating of quaternary ammonium salts or an ultrashort antimicrobial lipopeptide $\dagger$}

\author{
Tal Shalev, ${ }^{a}$ Anna Gopin, ${ }^{a}$ Michael Bauer, ${ }^{b}$ Robert W. Stark ${ }^{b c d}$ and Shai Rahimipour ${ }^{* a}$ \\ Received 16th August 2011, Accepted 21st November 2011 \\ DOI: 10.1039/c1jm13994k
}

\begin{abstract}
Bacterial fouling on surfaces significantly increases the resistance of bacteria toward antibiotics, which leads to medical complications and a corresponding financial burden. Here, we report on a general and robust technique for facile modification of various surfaces with different antibacterial agents. Our approach in this study was inspired by the strong adhesion of mussel adhesion proteins (MAPs) to many types of surfaces, including metals, polymers, and inorganic materials. Thus, glass and polymeric slides were dip-coated with dopamine, as a MAP mimic, and the resulting surfaces were characterized. The reactivity of dopamine-coated surfaces toward nucleophilic addition was then confirmed by reacting them with fluorescent probes containing either a free amino or a free thiol group. Laser scanning confocal microscopy (LSCM), X-ray photoelectron spectroscopy (XPS), confocal Raman microscopy, matrix-assisted laser desorption/ionization-time of flight (MALDI-TOF) mass spectroscopy, and cyclic voltammetry studies collectively suggested that the probes had covalently attached to the surfaces. Fabrication of dopamine-coated surfaces with an antibacterial quaternary amine or an ultrashort lipopeptide analog generated surfaces that effectively kill Escherichia coli and Staphylococcus aureus cells on contact. Moreover, minimal leaching of the fabricated agent was detected after prolonged incubation. This technique could be further developed to a "paint-like" or selfassembling monolayer-like procedure for the preparation of antibacterial surfaces on various materials.
\end{abstract}

\section{Introduction}

The increasing prevalence of bacterial infections in the recent years has led to an urgent demand for antibacterial surfaces. In particular, nosocomial infection and bacterial fouling on different surfaces have been reported to be a major cause of mortality, and they significantly increase the resistance of the bacteria toward antibiotics. ${ }^{1-4}$ Antibacterial materials find various uses in a wide variety of medical, industrial, public health, and environmental settings. They are particularly desirable in tissue regenerative applications, such as various implants or indwelling catheters, self-disinfecting surfaces, marine antifouling technologies, or devices that are in close contact with the human body. ${ }^{5-8}$

\footnotetext{
${ }^{a}$ Department of Chemistry, Bar-Ilan University, Ramat-Gan, 52900, Israel. E-mail: rahimis@biu.ac.il

${ }^{b}$ Center for Nanoscience CeNS, Ludwig-Maximilians-Universität Munchen, Munchen, 80799, Germany

'Department of Materials and Geosciences, Technische Universität Darmstadt, Darmstadt, 64287, Germany

${ }^{d}$ Center of Smart Interfaces, Technische Universität Darmstadt, Darmstadt, 64287, Germany

$\dagger$ Electronic supplementary information (ESI) available. See DOI: $10.1039 / \mathrm{cljm} 13994 \mathrm{k}$
}

Several approaches have been explored to prevent the attachment and growth of bacteria on surfaces. Antimicrobial surfaces have been generated via the incorporation of active biocides into the material or their adsorption onto the surface to allow their slow release into the near surroundings. ${ }^{9-12}$ However, their short-term antibacterial effect and the leaching of antibacterial agents with potential environmental toxicity limit their application. ${ }^{5}$ Furthermore, the release of the biocides into the environment increases the likelihood of bacterial resistance.

Materials with antibacterial activity can also be obtained by covalent attachment of the antibacterial agents to the surfaces. ${ }^{13-16}$ It is apparent, however, that the surfaces of the materials should be chemically modified before their reaction with the biocides. ${ }^{13,15}$ Many biomaterials, such as plastics, metals, and ceramics, have inert surfaces that required harsh chemical conditions or multiple chemical steps for their preactivation, which may limit scale-up processing. Additionally, the chemical transformations frequently require organic solvents that are not always compatible with the biomaterials. Therefore, there is an immediate need for the development of a general and bio-friendly "paint-like" procedure for the immobilization of antibacterial agents onto the surface of various materials. ${ }^{12,17}$

Mussels secrete adhesion proteins to tightly attach themselves to wet surfaces. The adhesive properties of mussel adhesion 
protein (MAP) are believed to be related to the overexpression of L-3,4-dihydroxyphenylalanine (DOPA) and lysine residues that are in close proximity with the substrate interface. ${ }^{18}$ Studies have shown that DOPA and other catechols can mimic the adhesive properties of MAP and be used as potent binding agents for coating inorganic surfaces. ${ }^{19,20}$ More recently, Messersmith et al. have reported that dopamine exerts the full adhesive properties of MAP and could be deposited as a thin adherent polymer film on different material surfaces, including metals, polymers, and inorganic materials. Moreover, the modified surfaces were shown to be versatile substrates for further ad-layer deposition of various compounds. ${ }^{21-23}$ The reactivity of the dopamine-coated surfaces is related to the formation of polydihydroxyindole (polydopamine; PDA) as a thin layer, which under oxidative (e.g. basic) conditions can oxidize to the corresponding polyorthoquinoneindole. The resulting film can subsequently interact covalently with various compounds via Schiff-base reactions (amine containing molecules) or Michael type reactions (amine and thiol containing molecules). ${ }^{21,22}$

The interfacial adhesion property of PDA coatings has been widely exploited to introduce new functionalities to the materials for new applications. ${ }^{24}$ For example, immobilization of vascular endothelial growth factor (VEGF) onto PDA-coated titanium alloy enhances microvascular endothelial cell attachment and proliferation, and induces differentiation of human mesenchymal stem cells into endothelial cells. ${ }^{25}$ PDA coating has been also used to anchor silver nanoparticles or lipase enzyme onto ferromagnetic nanoparticles $\left(\mathrm{Fe}_{2} \mathrm{O}_{3}\right)$ to afford particles with antibacterial or lipase activity that can be isolated from the medium by magnetic separation. ${ }^{26,27}$ PDA-coated surfaces immobilized by an initiator were also shown to be effective substrates to initiate atom transfer radical polymerization (ATRP) reaction to introduce polymer brushes into the surfaces. ${ }^{28,29}$

In this study, we utilized the adhesive properties of PDA to modify the surface of various materials with a quaternary ammonium salt (QAS) or an antimicrobial peptide (AMP) and so convert them to antibacterial surfaces. Cationic antimicrobials, such as QAS and AMP, are among the bactericides commonly used for the preparation of antibacterial surfaces. ${ }^{13,30,31}$ The mechanism of action of these molecules involves increased membrane permeation and the loss of membrane integrity. ${ }^{32}$ Membrane active antibacterial agents are ideal for surface modification since they can exhibit biological activity without penetrating the cell membrane. Moreover, the unique structure of these agents and their fast killing mechanism may significantly limit the development of drug resistant bacteria, which commonly develop in response to classic antibacterial and antifouling agents.

\section{Experimental section}

All chemicals and reagents were of analytical grade. AM-Rink amide resin, 9-fluorenylmethoxycarbonyl (Fmoc) protected amino-acid derivatives, and all other reagents for solid-phase peptide synthesis were purchased either from Novabiochem (San Diego, CA) or GL Biochem (Shanghai, China) and used as received. Unless otherwise stated, all other chemicals were obtained from Sigma-Aldrich (Rehovot, Israel).

\section{Peptides synthesis}

Peptides were synthesized manually by solid-phase peptide synthesis, employing the common Fmoc strategy and using an AM-Rink Amide MBHA resin (see ESI $\dagger$ ).

\section{Surface modification}

Polydopamine coating. The surfaces were coated with dopamine as described by Messersmith et al. ${ }^{21}$ Briefly, glass, polyolefine (Thermanox ${ }^{\circledR}$, Menzel-Gläser, Germany) and polystyrene (Nunc, Denmark) slides were cleaned ultrasonically in 2-propanol for 10 minutes before the coating. The slides were then dip-coated into a stirred solution of dopamine $\left(2 \mathrm{mg} \mathrm{mL}^{-1}\right.$, $10 \mathrm{mM}$ Tris- $\mathrm{HCl}, \mathrm{pH} 8.5$ ) for $14 \mathrm{~h}$. Following the incubation of the surfaces with dopamine, the color of the slides changed from colorless to a dark brown. The coated surfaces were rinsed with water and dried by $\mathrm{N}_{2}$ gas before storage or treated immediately for ad-layer formation.

\section{Fabrication of the PDA-coated surfaces}

For ad-layer deposition of fluorescent probe $\mathbf{1}$ and QASs 3 and $\mathbf{4}$, $0.3 \mathrm{mM}$ solutions of $\mathbf{1}, \mathbf{3}$, and $\mathbf{4}$ were prepared in DCM. Tris(2carboxyethyl) phosphine (TCEP) (2 eq.) was added to the solutions of $\mathbf{1}$ and $\mathbf{3}$ to prevent the formation of disulfide bonds and to reduce those already generated. The solutions were then added to PDA-coated surfaces in pressure tubes, after which triethylamine (TEA) was added (final concentration $10 \mathrm{mM}$ ). The tubes were sealed and heated at $50{ }^{\circ} \mathrm{C}$ for 3 days. Next, the surfaces were rinsed with DCM and ethanol and dried in a $\mathrm{N}_{2}$ stream.

For fabrication of water-soluble substrates 2, 5, and $\mathbf{6}$, the compounds were dissolved in Tris buffer $(10 \mathrm{mM}, \mathrm{pH} 8.0)$ to afford a $1 \mathrm{mM}$ final concentration. TCEP (2 eq.) was added to the solution of $\mathbf{5}$ to prevent disulfide formation. PDA-coated slides were then placed into a 24 well plate containing the above solutions and sealed with a multiwell aluminium sealing film (Excel Scientific, CA, USA). After 3 days of incubation at $50{ }^{\circ} \mathrm{C}$, the surfaces were rinsed with water and ethanol, and dried in a $\mathrm{N}_{2}$ stream.

\section{Surface characterization}

Atomic force microscopy (AFM). The surface morphology of the surfaces was evaluated by a Nanoscope V Multimode scanning probe microscope (Digital Instruments, Santa Barbara, CA). All images were obtained in the tapping mode with a single Point Probe ${ }^{\circledR}$ Plus Non-Contact/Tapping Mode-Long Cantilever (PPP-NCL) silicon probe (force constant of 21-98 $\mathrm{N} \mathrm{m}^{-1}$, Digital Instruments, Santa Barbara, CA). The images were captured in the retrace direction at a scan rate of $1 \mathrm{~Hz}$. Scan size was $5 \times 5 \mu \mathrm{m}$ and image resolution was 256 samples per line. The roughness was determined by an analysis of the AFM height images employing Nanoscope Software Version 7.3.

X-Ray photoelectron spectroscopy (XPS). Analyses were performed with a Kratos AXIS-HS spectrometer (Manchester, UK) using a monochromatized Al-K $\alpha$ source and scans at $75-150 \mathrm{~W}$. All data acquisitions were performed in a hybrid mode (using electrostatic and magnetic lenses) with detection pass energies of 
40-80 eV. All XPS measurements were carried out at room temperature, under vacuum conditions $\left(\sim 10^{-9}\right.$ Torr $)$. The spectra were acquired with an electron flood gun for charge neutralization, and data processing was preformed with Vision 2.1 software ("Kratos"). Vision 2.1 sensitivity factors were used for quantification. The $\mathrm{C} 1 \mathrm{~s}$ peak was set to $285 \mathrm{eV}$ for energy calibration.

MALDI-TOF MS. Mass spectroscopy analyses were performed using a Bruker Autoflex III MALDI TOF/TOF spectrometer (Bruker, Germany) with a $20 \mathrm{kV}$ accelerating voltage in positive reflector mode. Slides were introduced to a special MALDI plate and directly analyzed.

\section{In vitro antibacterial activity}

Compounds were assayed for their antibacterial activity according to the NCCLS Guidelines ${ }^{33}$ with minor changes. Briefly, aliquots $(30 \mu \mathrm{L})$ of $S$. aureus (FRF1169) or E. coli (C600), at concentrations of $1.6 \times 10^{6} \mathrm{cfu} \mathrm{mL}^{-1}$ in the culture medium, were added to $70 \mu \mathrm{L}$ of twofold serial dilutions of the peptides (prepared from a stock solution of $1 \mathrm{mM}$ ) in lysogeny broth (LB). Norfloxacin with broad-spectrum antibiotic activity was used as a positive control. The plates were incubated for $20 \mathrm{~h}$ at $37{ }^{\circ} \mathrm{C}$ with gentle shaking and the inhibition of growth was determined by measuring the absorbance at $600 \mathrm{~nm}$, using a plate reader (Infinite M-200, Tecan, Switzerland). The antibacterial activity was expressed as the minimal inhibitory concentration (MIC) at which no growth of the bacteria was observed. The experiments were performed in duplicates and repeated three times.

\section{Antimicrobial susceptibility determination of the fabricated surfaces}

A suspension of $S$. aureus or E. coli $(10 \mu \mathrm{L})$ in LB containing $30 \%$ glycerol was added to $5 \mathrm{~mL}$ of LB broth in a sterile $50 \mathrm{~mL}$ tube. The suspension was then incubated at $37^{\circ} \mathrm{C}$ with shaking at 200 $\mathrm{rpm}$ for $16 \mathrm{~h}$. After centrifugation $(2700 \mathrm{rpm}, 10 \mathrm{~min})$, the cells were washed with distilled water and resuspended at a concentration of $1 \times 10^{6}$ cells ml $^{-1}\left(5 \times 10^{7}\right.$ in the case of $E$. coli $)$. The bacterial suspensions were then sprayed in a fume hood onto the sterile surfaces of the fabricated slides using a commercial chromatography sprayer (spray rate $\approx 10 \mathrm{~mL} \min ^{-1}$ ). After drying the slides for 2-5 min under air, the slides were flipped over into growth agar (1.5\% agar in LB broth) in a pre-prepared Petri dish. The Petri dish was closed, sealed, and incubated at $37^{\circ} \mathrm{C}$ overnight, and the colonies were then counted.

\section{Results and discussion}

We dip-coated glass, polystyrene, Thermanox ${ }^{\circledR}$ polyolefine, and indium tin oxide-(ITO-) coated glass with a thin layer of PDA by immersing them in a basic solution of aqueous dopamine $(2 \mathrm{mg}$ $\mathrm{mL}^{-1}$ in Tris buffer, $\mathrm{pH}$ 8.5). Dopamine polymerization in basic solution occurs via oxidation and intramolecular rearrangement and produces a melanin-like structure (Fig. S1, see ESI $\dagger$ ). During the incubation of the surfaces in the dopamine solution, the color of the slides gradually changed from transparent to dark brown.

The formation of a PDA coating was confirmed by atomic force microscopy (AFM, Fig. 1A and $\mathrm{S} 2 \dagger$ ), matrix-assisted laser

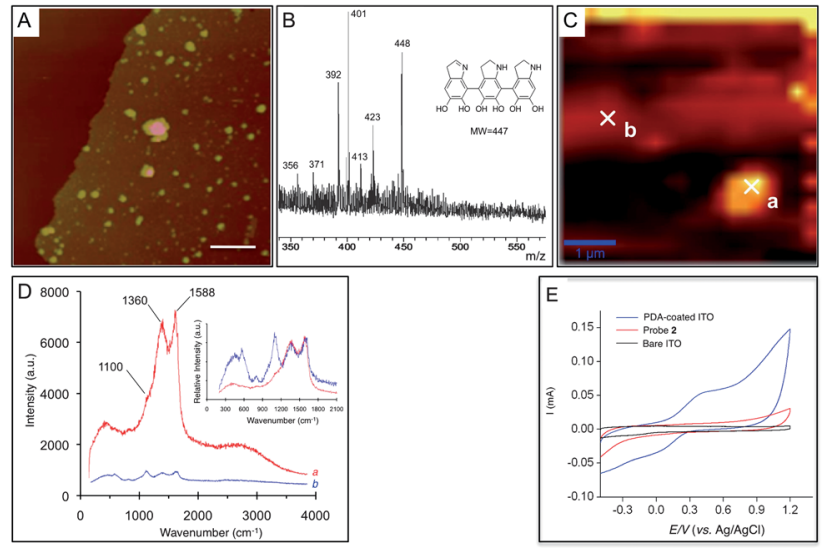

Fig. 1 Surface analysis of polydopamine-(PDA-) coated glass surfaces. (A) 3D topographic AFM scan of glass partially coated with PDA and (B) the corresponding MALDI-TOF spectrum of the PDA-coated glass surface. (C) Confocal optical micrograph of the coated surface and (D) corresponding Raman spectrum of the spots that are marked on the confocal micrograph (C). Spot (a) represents a polydopamine aggregate that is formed on the PDA-coated background (b). (E) Cyclic voltammograms of bare ITO, PDA-coated ITO, and PDA-coated ITO that was reacted with AMP 6. Measurements were carried out in aqueous phosphate buffered saline solution (100 mM, pH 7.4).

desorption/ionization-time of flight (MALDI-TOF) mass spectroscopy (Fig. 1B), and confocal Raman microscopy (Fig. 1C and D). The AFM studies of PDA-coated glass slides suggested the generation of a layer with a thickness of 25-30 nm whose roughness was significantly increased by the precipitation of PDA aggregates on its surface (Fig. S2, ESI $\dagger$ ). The mass spectroscopy of PDA-coated glass slides showed a molecular peak at $448 \mathrm{~m} / \mathrm{z}$ that, according to the obtained fragmentation pattern, represents the 5,6-dihydroxyindole trimer. ${ }^{21}$ Confocal Raman microscopy demonstrated the appearance of two signals at 1360 and $1588 \mathrm{~cm}^{-1}$, which are attributed to catechol $\mathrm{C}=\mathrm{O}$ stretching vibrations. ${ }^{34}$ Since the PDA coating is suspected to be redox active, we also analyzed the PDA-coated ITO surface by cyclic voltammetry (Fig. 1E). The voltammogram showed a quasireversible electron transfer step at $0.37 \mathrm{~V}$ (vs. $\mathrm{Ag} / \mathrm{AgCl})$, which closely resembles that of dopamine in buffered solution. ${ }^{35,36}$ The redox step most likely represents a two-electron, two-proton process oxidation of a polymerized dopamine film to form the corresponding reactive ortho-quinone derivative.

To confirm the chemical reactivity of the PDA-coated surfaces toward nucleophilic addition (Michael-type reaction), fluorescent probes $\mathbf{1}$ and $\mathbf{2}$ were synthesized by solid-phase peptide synthetic methods, utilizing the common Fmoc procedure (Scheme 1). Probe 1 contained a free thiol group, which is a better nucleophile than the free amino group present in probe $\mathbf{2}$. However, probe 2 included a polyethylene glycol-(PEG-) like spacer that enhances the solubility of the probe in aqueous solutions, which is necessary for its reaction with the polymeric substrate (polystyrene or polyolefine). The PEG spacer can also provide the surfaces with the properties required to resist the accumulation of proteins and cells and so suppress the attachment of live bacteria to dead cells or cell material.

Probe 1 was then reacted in dichloromethane with PDAcoated glass in a pressure tube in the presence of 


$$
\text { (ns) }
$$

CQ10 analog

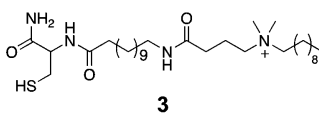

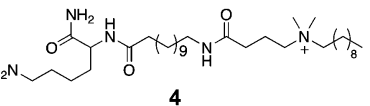

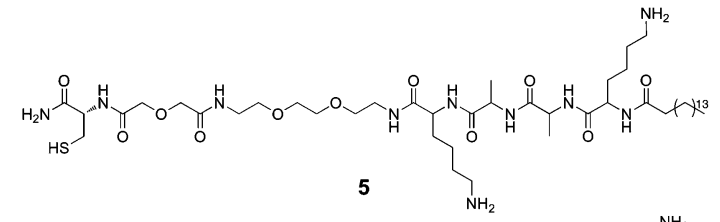

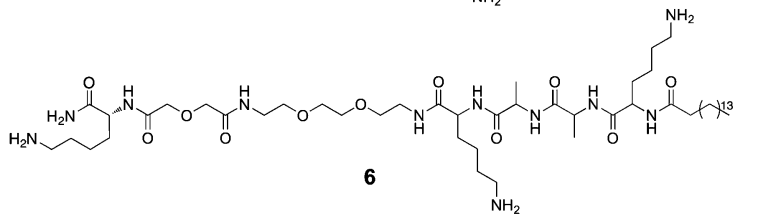

Scheme 1 Chemical structures of the fluorescent probes $\mathbf{1}$ and $\mathbf{2}$, quaternary ammonium salts $\mathbf{3}$ and $\mathbf{4}$, and ultrashort antimicrobial lipopeptides 5 and $\mathbf{6}$ that were incorporated into polydopamine-coated surfaces.

diisopropylethylamine (DIPEA) for 3 days at $50{ }^{\circ} \mathrm{C}$. In order to minimize the oxidation of 1 to the corresponding disulfide, Tris (2-carboxyethyl)phosphine (TCEP) was added to the solution prior to addition of the mixture to the coated glass. Probe $\mathbf{2}$ was reacted with the surfaces through incubation in $10 \mathrm{mM}$ Tris buffer ( $\mathrm{pH}$ 8.5) with PDA-coated glass, polystyrene, or polyolefine slides at $50{ }^{\circ} \mathrm{C}$ for 3 days. The slides were then extensively washed with either dichloromethane or water to remove the unreacted probes, rinsed with absolute ethanol, and dried in a stream of $\mathrm{N}_{2}$.

The surfaces were then characterized by laser scanning confocal microscopy (LSCM), fluorescent spectrometry, cyclic voltammetry, X-ray photoelectron spectroscopy (XPS), and MALDI-TOF mass spectrometry (Fig. 2). The LSCM and fluorescent studies demonstrated a significantly higher fluorescent signal on the treated PDA surfaces as compared to nontreated PDA surfaces, suggesting the presence of probes $\mathbf{1}$ and $\mathbf{2}$ on the PDA-coated surfaces. The extent of surface modification with 2 was also determined by CV. Fabricating 2 onto PDAcoated ITO surfaces caused the PDA signal to disappear in the tested range (Fig. 1E). This can be rationalized by the significant increase in the redox potential of amino conjugated PDA as compared to unmodified PDA. ${ }^{37}$ The disappearance of the CV signal also suggests that probe $\mathbf{2}$ reacted with high fidelity with the PDA surface. XPS analysis of the PDA surfaces fabricated with 1 revealed the presence of sulfur on the surfaces $(160.5 \mathrm{eV}$ for S 2p; Fig. 2D), while analysis of the surfaces fabricated with 2 gave ambiguous results. On the other hand, the MALDI-TOF analysis of 2 showed a peak at $660.356 \mathrm{Da}$, which corresponds to the T2 fraction of $\mathbf{2}$ following the cleavage of the NBD group from the terminal amine (Fig. 2E). Cleavage of the NBD group from polypeptides was also observed in other studies. ${ }^{38}$ Remarkably, mass analysis of $\mathbf{1}$ under the same conditions did not yield the corresponding molecular mass, suggesting the more
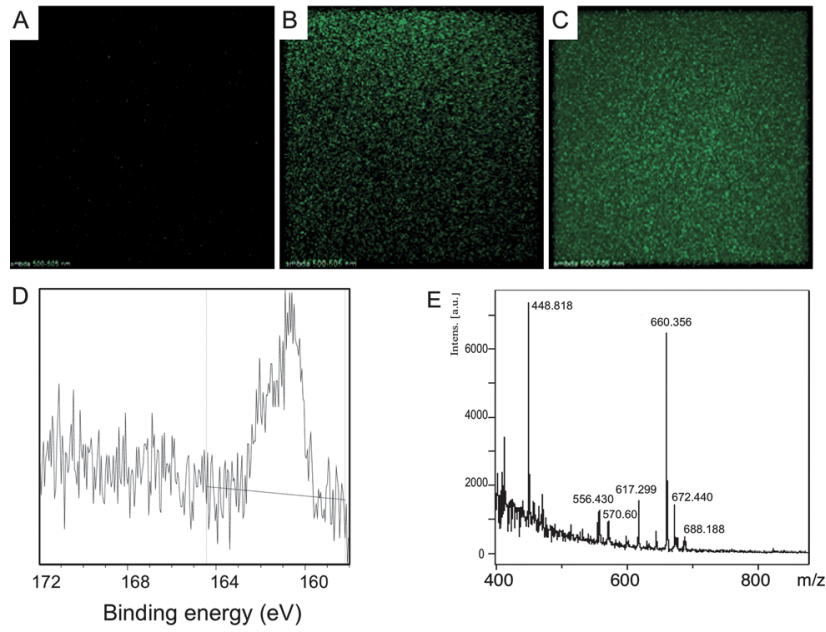

Fig. 2 Surface analysis of polydopamine-(PDA-) coated glass fabricated with fluorescent probe $\mathbf{1}$ (which contains a free thiol group) and probe $\mathbf{2}$ (which contains a free amine group and a polyethylene glycol spacer). (A) LSCM analysis of untreated PDA-coated glass and of PDA surfaces treated with (B) 1 and(C) 2. The surfaces were excited at $458 \mathrm{~nm}$ and emission was recorded at 500-510 $\mathrm{nm}$. (D) An XPS analysis of the glass surfaces fabricated with probe 1 showing a characteristic sulfur $2 \mathrm{p}$ peak at $160.5 \mathrm{eV}$. (E) MALDI-TOF analysis of the PDA-coated glass surface modified with probe 2 .

reversible nature of the bond between $\mathbf{2}$ and the quinonic part of PDA under MALDI-TOF mass spectroscopy conditions.

In order to investigate whether the immobilized probes leach from the surface, PDA-coated glass slides fabricated with probe 2 were incubated for 3-5 days in PBS solution and the extent of 2 released into the medium was determined by fluorescent and HPLC analyses. No detectable release of $\mathbf{2}$ was evident in the cultured medium, suggesting the strong binding of $\mathbf{2}$ to the PDA surfaces. $^{22}$

The reactivity of the PDA-coated surfaces toward nucleophiles under mild reaction conditions and the tight binding of the immobilized compounds to the PDA-coated surfaces prompted us to utilize this method for a facile, one-step generation of antimicrobial surfaces. Two analogs of well-known antibacterial dialkyldimethylammonium $\operatorname{salt}^{39}$ (3 and 4, Scheme 1) and two derivatives of ultrashort antibacterial lipopeptides $(5 \text { and } 6)^{40}$ were synthesized. All the analogs bore a spacer containing either a terminal free thiol or amine through which they were bound onto the PDA-coated surfaces. Dialkyldimethylammonium salts 3 and 4 were based on CQ10 (Scheme 1), which is a QAS that exhibits potent antibacterial and antifungal activity when immobilized onto a gold nanoparticle surface. ${ }^{39}$ Lipopeptides 5 and 6 were derived from the AMP peptide $\mathrm{KA} A \mathrm{~K}$ (where $A$ stands for D-Ala) containing a $\mathrm{C} 16$ alkyl chain on its $N$-terminal (C16-KA $A \mathrm{~K})$. Shai et al. have recently reported that ultrashort lipopeptides, such as $\mathrm{C} 16-\mathrm{KA} A \mathrm{~K}$, exhibit potent activity against bacteria, fungi, and yeast. ${ }^{40}$ Unlike QASs, which are generally toxic to all types of cells, the ultrashort lipopeptides exhibit low hemolytic activity, which suggests that they have low toxicity toward mammalian cells. ${ }^{40}$ A polyethylene glycol-(PEG-) like spacer was introduced onto the $C$-terminal of the original lipopeptide followed by the addition of a Cys or a Lys to yield 
lipopeptides 5 and $\mathbf{6}$, respectively. The PEG moiety was incorporated to enhance water solubility, induce molecular flexibility, and introduce antifouling activity to repel dead bacteria from the surface. All the derivatives were synthesized on solid supports, employing an Fmoc solid-phase peptide synthesis protocol, and were characterized by mass spectroscopy.

To validate the antibacterial activity of the new derivatives against Escherichia coli (E. coli) and Staphylococcus aureus ( $S$. aureus), the microdilution antimicrobial susceptibility test was performed. ${ }^{33}$ Parent C16-KA $A \mathrm{~K}$ and CQ10 were used as the controls. Table 1 shows the in vitro antibacterial activities of the AMP and QAS derivatives tested against the Gram-positive and the Gram-negative bacteria. The results obtained with QAS analog 3 suggest that substitution of the terminal thiol group in CQ10 with a Cys group has a minimal effect on antibacterial activity. Incorporation of a Lys to this position in $\mathbf{4}$, however, completely abolished the activity. Unexpectedly, modification of the $C$-terminal of the lipopeptide, C16-KA $A \mathrm{~K}$, with a PEGylated Cys derivative substantially decreased the antibacterial activity of 5 toward both strains, while incorporation of a PEGylated Lys to this position induced $E$. coli selectivity into the antibacterial activity of $\mathbf{6}$.

Next, we tested the reactivity of the PDA-coated glass surfaces toward the active QAS analog 3 and AMPs 5 and 6. PDA-coated glass slides were reacted with QAS 3 in DCM or with AMPs 5 and $\mathbf{6}$ in Tris buffer, as described. The surfaces were then characterized either by XPS (3 and 5) or MALDI-TOF mass spectroscopy (6). The XPS analysis of the surfaces fabricated with 3 and 5 demonstrated the appearance of thiol signals at 163.8 and $163.4 \mathrm{eV}$, respectively, while the mass spectroscopy measurement of the surface reacted with 6 revealed a peak at an $\mathrm{m} / \mathrm{z}$ of 1050.793 corresponding to the $[\mathrm{M}+\mathrm{Na}]^{+}$ion (Fig. 3). These data confirm the existence of probes $\mathbf{3}, \mathbf{5}$, and $\mathbf{6}$ on the PDA-coated surfaces.

To determine whether the modified surfaces exhibit antibacterial activity on contact, the method of Tiller et al. was employed. ${ }^{13}$ The method mimics the deposition of airborne bacteria on various surfaces, which is the major mode of transmission for bacterial infections. Overnight-grown $E$. coli and $S$. aureus bacteria were suspended in distilled water and sprayed onto the sterile surface of the fabricated slides. The slides were dried and then flipped-over on pre-prepared growth agar. PDAcoated glass surfaces sprayed with the same bacterial suspensions served as controls. Bacteria growth was then assessed by

Table 1 In vitro antimicrobial activity of the synthesized quaternary ammonium salt-based (QAS-based) and antimicrobial peptide-based (AMP-based) compounds

$\mathrm{MIC}^{a} / \mu \mathrm{mol} \mathrm{L}{ }^{-1}$

\begin{tabular}{lrlllll}
\hline & \multicolumn{2}{c}{ Compound } & & & \\
\cline { 2 - 7 } Strain & $\mathbf{3}$ & $\mathbf{4}$ & $\mathbf{5}$ & $\mathbf{6}$ & $\mathbf{C Q 1 0}^{b}$ & ${\mathbf{C 1 6 - K A} \boldsymbol{A} \mathbf{K}^{c}}^{-1}$ \\
\hline E. coli & 200 & 500 & 400 & 15 & N/A & 30 \\
S. aureus & 30 & $>500$ & 200 & 125 & 17.5 & 30
\end{tabular}

${ }^{a}$ Minimum inhibitory concentration. ${ }^{b}$ QAS control for compounds 3 and $4 .{ }^{16}{ }^{c}$ AMP-based ultrashort lipopeptide control for compounds 5 and $6 .{ }^{17}$
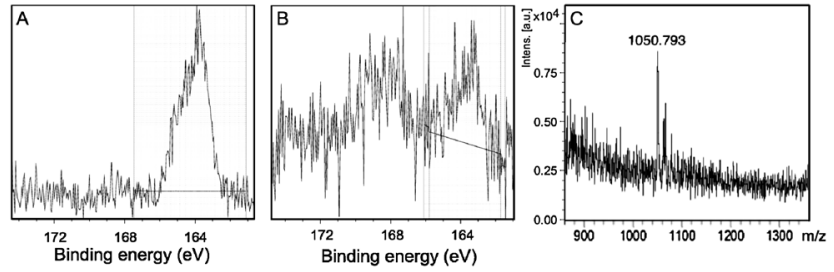

Fig. 3 Surface analysis of polydopamine-(PDA-) coated glass fabricated with quaternary ammonium salt (QAS) analog 3 or antimicrobial peptides (AMPs) 5 or 6. (A) XPS analysis of the PDA-coated surfaces after their reaction with QAS 3 and (B) AMP 5. Both surfaces exhibited characteristic sulfur 2p peaks (at 163.8 and $163.4 \mathrm{eV}$, respectively) derived from QAS 3 and AMP 5. (C) MALDI-TOF analysis of the PDA surfaces reacted with AMP 6. The numbered peak corresponds to the $[\mathrm{M}+\mathrm{Na}]^{+}$ ion, which is attributable to AMP 6.

counting the bacterial colonies after incubation overnight. Fig. 4 shows colonies of $E$. coli and $S$. aureus that were grown on PDAcoated glass slides fabricated with QAS 3 and AMPs 5 and 6 after being sprayed with the bacterial suspensions and incubated overnight. A significantly lower number of bacterial colonies was evident in the case of the fabricated surfaces as compared to the untreated surfaces. The quantitative changes in bacterial numbers following their incubation with the surfaces are summarized in Fig. 4B. The data suggest that surfaces treated with $\mathbf{3}$ or $\mathbf{6}$ were very effective in reducing the number of viable bacterial cells. Treatment with 3 was most effective in this respect, with $>88 \%$ reduction in bacterial growth for both strains (Fig. 4). Remarkably, QAS 3 and AMP 6 also demonstrated the highest antibacterial activity in MIC experiments, although the MIC of 3 toward E. coli was significantly higher than toward S. aureus (Table 1).

Having demonstrated the high efficacy and robustness of the PDA-coating methodology to generate surfaces that are reactive toward nucleophilic reaction, and the high surface antibacterial activity of immobilized QAS 3 and AMP 6, we next tested the versatility of this approach with respect to other polymeric surfaces. Synthetic polymers are widely used in diverse applications that involve human contact. For example, polymeric materials are often used in food packaging, textiles, and biomedical applications, such as implants and catheters, which can easily be colonized by bacteria and fungi, causing severe infections and transmitting diseases. ${ }^{\mathbf{8} 30}$ Moreover, they are relatively chemically inert and so frequently require aggressive treatment to alter their physical properties before they can be modified at their surface. ${ }^{41-43}$ Therefore, surface modification of polymeric materials, and particularly of polystyrene and polyolefine surfaces, is of major interest. ${ }^{16,44,45}$ To this end, the surfaces of polystyrene and polyolefine slides were dip-coated with PDA and water-soluble AMP 6 was covalently bound to the resulting surfaces. The antibacterial activity of the modified surfaces toward airborne $S$. aureus was then determined and compared to that of parent PDA-coated surfaces. We found that the ultrashort AMP 6 preserves its ability to kill $S$. aureus when fabricated on both polymers (Fig. S3, ESI $\dagger$ ). Remarkably, the antibacterial activity of $\mathbf{6}$ was significantly better ( $>96 \%$ bacterial killing) when it was immobilized on either polymer surface than when it was bound to 
A

E. Coli
Control

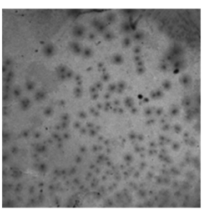

S. aureus

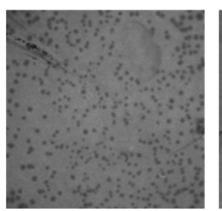

3
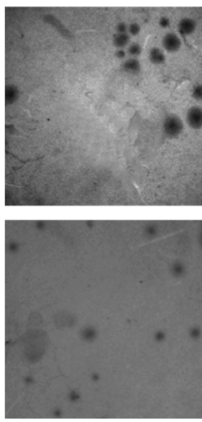

5

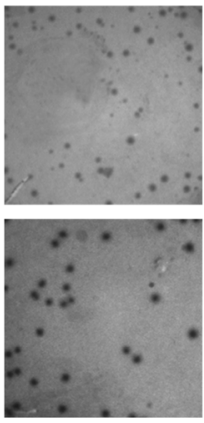

6

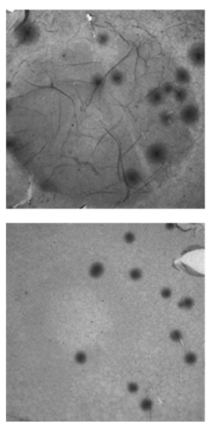

B

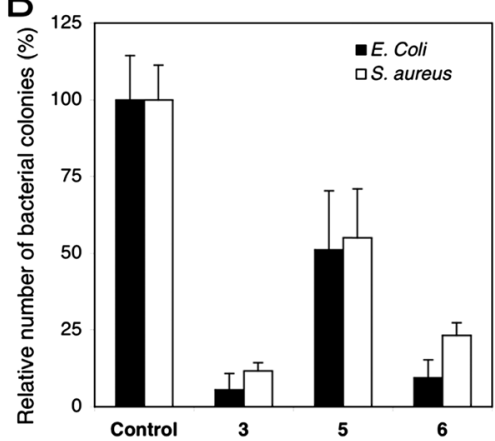

Fig. 4 (A) Representative photographs of E. coli and S. aureus colonies grown on polydopamine-(PDA-) coated glass slides (control) or on PDAcoated glasses modified with quaternary ammonium salt (QAS) 3 or antimicrobial peptides (AMPs) 5 or 6 . PDA-coated glass slides were fabricated with QAS 3 or AMPs 5 or 6 and then sprayed with an aqueous suspension of E. coli $\left(\sim 5 \times 10^{7}\right.$ cells per ml of distilled water) or $S$. aureus cells $\left(\sim 10^{6}\right.$ cells per $\mathrm{ml}$ of distilled water). The surfaces were then air dried and incubated under $1.5 \%$ agar in a bacterial growth medium at $37^{\circ} \mathrm{C}$ overnight. (B) The relative growth of E. coli and $S$. aureus colonies on the surface of the fabricated PDA-coated glass slides. Untreated PDA-coated glass slides were used as a control $(100 \%)$. The experiments were performed in quadruplicate, and the error bars indicate the standard deviations from the mean values.

a PDA-coated glass surface. This may suggest that better PDA coating occurred with the polymeric surfaces or that there was a more efficient interaction between AMP and these PDAcoated surfaces.

To further confirm that immobilized AMP 6 cannot leach from the PDA-coated surfaces, we examined the growth of the bacteria in the immediate proximity of the fabricated PDAcoated glass slides. We assumed that if the fabricated AMP 6 leached from the surface into the near environment, it should inhibit the growth of the bacteria in close proximity to the coated glass. After spraying $S$. aureus on the surface, the slides were layered over agar and incubated overnight. While only a few bacterial colonies grew on the surface of the fabricated slides, considerable bacterial growth was evident in close proximity to the slides (Fig. S4, ESI $\dagger$ ). This suggests that the immobilized AMP does not leach from the slides into the near environment and that the bacteria are killed only upon their contact with the surface of the slides. ${ }^{46}$

\section{Conclusion}

We have demonstrated a facile, versatile, and bio-inspired method to fabricate different surfaces with antibacterial quaternary ammonium salts and novel ultrashort antimicrobial lipopeptides. The fast acting mechanism of these agents together with their broad antimicrobial activity may reduce the development of antibiotic resistance. We showed that the antibacterial activity of the new analogs was not adversely affected by their immobilization onto different surfaces, and the fabricated surfaces effectively kill airborne $E$. coli and $S$. aureus on contact. Moreover, no leaching of the active antibacterial agents into the near environment was detected. The high selectivity of the ultrashort lipopeptides for bacterial cells coupled with their minimal toxicity to mammalian cells may enable the utilization of this method for various biomedical and even food packing purposes. Moreover, we believe that this methodology could be further developed to enable the fabrication of large antibacterial surfaces by means of a "paint-like" process.

\section{Acknowledgements}

We are grateful to the German-Israel Foundation for their financial support of this study. We also thank Dr. David Donovan of the Animal Biosciences and Biotechnology Lab, ANRI, ARS, USDA, Beltsville, MD, USA, for helpful discussions and suggestions. We would also like to acknowledge Dr Yosef Gofer and Dr Rachel Perski for their technical assistance in selected aspects of this work.

\section{References}

1 L. L. Leape, T. A. Brennan, N. Laird, A. G. Lawthers, A. R. Localio, B. A. Barnes, L. Hebert, J. P. Newhouse, P. C. Weiler and H. Hiatt, $N$. Engl. J. Med., 1991, 324, 377-384.

2 P. S. Stewart and J. W. Costerton, Lancet, 2001, 358, 135-138.

3 R. M. Klevens, J. R. Edwards, C. L. Richards, Jr, T. C. Horan, R. P. Gaynes, D. A. Pollock and D. M. Cardo, Public Health Rep., $2007,122,160-166$.

4 J. Chandra, G. Zhou and M. A. Ghannoum, Curr. Drug Targets, 2005, 6, 887-894.

5 H. Murata, R. R. Koepsel, K. Matyjaszewski and A. J. Russell, Biomaterials, 2007, 28, 4870-4879.

6 J. C. Tiller, C. J. Liao, K. Lewis and A. M. Klibanov, Proc. Natl. Acad. Sci. U. S. A., 2001, 98, 5981-5985.

7 B. Jose, V. Antoci, Jr, A. R. Zeiger, E. Wickstrom and N. J. Hickok, Chem. Biol., 2005, 12, 1041-1048.

8 A. M. Klibanov, J. Mater. Chem., 2007, 17, 2479-2482.

9 J. M. Schierholz, L. J. Lucas, A. Rump and G. Pulverer, J. Hosp. Infect., 1998, 40, 257-262.

10 G. L. Woo, M. W. Mittelman and J. P. Santerre, Biomaterials, 2000, 21, 1235-1246.

11 R. Gottesman, S. Shukla, N. Perkas, L. A. Solovyov, Y. Nitzan and A. Gedanken, Langmuir, 2011, 27, 720-726.

12 A. Kumar, P. K. Vemula, P. M. Ajayan and G. John, Nat. Mater., 2008, 7, 236-241.

13 J. C. Tiller, C. J. Liao, K. Lewis and A. M. Klibanov, Proc. Natl. Acad. Sci. U. S. A., 2001, 98, 5981-5985.

14 M. Ignatova, S. Voccia, B. Gilbert, N. Markova, D. Cossement, R. Gouttebaron, R. Jerome and C. Jerome, Langmuir, 2006, 22, 255-262.

15 N. Aumsuwan, S. Heinhorst and M. W. Urban, Biomacromolecules, 2007, 8, 713-718.

16 J. Huang, H. Murata, R. R. Koepsel, A. J. Russell and K. Matyjaszewski, Biomacromolecules, 2007, 8, 1396-1399.

17 K. Mukherjee, J. J. Rivera and A. M. Klibanov, Appl. Biochem. Biotechnol., 2008, 151, 61-70. 
18 J. H. Waite, J. Biol. Chem., 1983, 258, 2911-2915.

19 M. Yu and T. J. Deming, Macromolecules, 1998, 31, 4739-4745.

20 J. Y. Wach, S. Bonazzi and K. Gademann, Angew. Chem., Int. Ed., 2008, 47, 7123-7126.

21 H. Lee, S. M. Dellatore, W. M. Miller and P. B. Messersmith, Science, 2007, 318, 426- 430.

22 H. Lee, N. F. Scherer and P. B. Messersmith, Proc. Natl. Acad. Sci. U. S. A., 2006, 103, 12999-13003.

23 H. Lee, J. Rho and P. B. Messersmith, Adv. Mater., 2009, 21, 431-434

24 Q. Ye, F. Zhou and W. M. Liu, Chem. Soc. Rev., 2011, 40, 4244-4258.

25 P. Chye Khoon, S. Zhilong, L. Tee Yong, N. Koon Gee and W. Wang, Biomaterials, 2010, 31, 1578-1585.

26 M. Sureshkumar, D. Y. Siswanto and C. K. Lee, J. Mater. Chem., 2010, 20, 6948-6955.

27 Y. H. Ren, J. G. Rivera, L. H. He, H. Kulkarni, D. K. Lee and P. B. Messersmith, BMC Biotechnol., 2011, 11, DOI:10.1186/14726750-11-63.

28 H. Y. Hu, B. Yu, Q. Ye, Y. S. Gu and F. Zhou, Carbon, 2010, 48, $2347-2353$

29 B. C. Zhu and S. Edmondson, Polymer, 2011, 52, 2141-2149.

30 L. Ferreira and A. Zumbuehl, J. Mater. Chem., 2009, 19, 7796-7806.

31 P. A. Fulmer, J. G. Lundin and J. H. Wynne, ACS Appl. Mater. Interfaces, 2010, 2, 1266-1270.

32 Z. Oren and Y. Shai, Biopolymers, 1998, 47, 451-463.

33 Methods for Dilution Antimicrobial Susceptibility Tests for Bacteria that Grow Aerobically, Approved standard NCCLS Document M7A4, Wayne, PA, 4th edn, vol. 17, January 1997.

34 S. H. Ku, J. S. Lee and C. B. Park, Langmuir, 2010, 26, 15104-15108.
35 P. Diaz, Y. Gimeno, P. Carro, S. Gonzalez, P. L. Schilardi, G. Benitez, R. C. Salvarezza and A. H. Creus, Langmuir, 2005, 21, 5924-5930.

36 S. Chen, K. Y. Tai and R. D. Webster, Chem.-Asian J., 2011, 6, 14921499.

37 G. Klopman and N. Doddapaneni, J. Phys. Chem., 1974, 78, 18201825.

38 G. G. Borisenko, A. A. Kapralov, V. A. Tyurin, A. Maeda, D. A. Stoyanovsky and V. E. Kagan, Biochemistry, 2008, 47, 13699-13710.

39 P. Thebault, E. Taffin de Givenchy, R. Levy, Y. Vandenberghe, F. Guittard and S. Geribaldi, Eur. J. Med. Chem., 2009, 44, 717724.

40 A. Makovitzki, D. Avrahami and Y. Shai, Proc. Natl. Acad. Sci. U. S. A., 2006, 103, 15997-16002.

41 H. S. Choi, V. V. Rybkin, V. A. Titov, T. G. Shikova and T. A. Ageeva, Surf. Coat. Technol., 2006, 200, 4479-4488.

42 K. Fujimoto, Y. Takebayashi, H. Inoue and Y. Ikada, J. Polym. Sci., Part A: Polym. Chem., 1993, 31, 1035-1043.

43 H. Y. Nie, M. J. Walzak, B. Berno and N. S. McIntyre, Appl. Surf. Sci., 1999, 144-145, 627-632.

44 S. Krishnan, R. J. Ward, A. Hexemer, K. E. Sohn, K. L. Lee, E. R. Angert, D. A. Fischer, E. J. Kramer and C. K. Ober, Langmuir, 2006, 22, 11255-11266.

45 J. M. Goddard and J. H. Hotchkiss, J. Food Prot., 2008, 71, 2042 2047.

46 Y. Endo, T. Tani and M. Kodama, Appl. Environ. Microbiol., 1987, 53, 2050-2055. 